\title{
Elastic constants from direct correlation functions in nematic liquid crystals: A computer simulation study
}

\author{
Nguyen Hoang Phuong, Guido Germano, and Friederike Schmid \\ Fakultät für Physik, Universität Bielefeld, 33501 Bielefeld, Germany
}

(Received 27 March 2001; accepted 27 July 2001)

\begin{abstract}
Density functional theories such as the Poniewierski-Stecki theory relate the elastic properties of nematic liquid crystals with their local liquid structure, i.e., with the direct correlation function (DCF) of the particles. We propose a way to determine the DCF in the nematic state from simulations without any approximations, taking into account the dependence of pair correlations on the orientation of the director explicitly. Using this scheme, we evaluate the Frank elastic constants $K_{11}, K_{22}$, and $K_{33}$ in a system of soft ellipsoids. The values are in good agreement with those obtained directly from an analysis of order fluctuations. Our method thus establishes a reliable way to calculate elastic constants from pair distributions in computer simulations. (C) 2001 American Institute of Physics. [DOI: 10.1063/1.1404388]
\end{abstract}

\section{INTRODUCTION}

Nematic liquid crystals are fluids of anisotropic particles, which are aligned preferentially along one direction. ${ }^{1,2}$ Their orientation is characterized by a director $\mathbf{n}$ of unit length, with physically identical states $\mathbf{n}$ and $-\mathbf{n}$. Since the long range orientational order breaks a continuous symmetry, the isotropy of space, there exist soft fluctuation modes-spatial variations of the director $\mathbf{n}(\mathbf{r})$ - which cost no energy in the infinite wavelength limit (i.e., the limit where $\mathbf{n}$ is rotated uniformly) and are otherwise penalized by elastic restoring forces. ${ }^{3,4}$ For symmetry reasons, the latter depend on only three material parameters at large finite wavelengths. ${ }^{1-6}$ They are described by an elastic free energy functional ${ }^{6}$

$$
\begin{aligned}
\mathcal{F}\{\mathbf{n}(\mathbf{r})\}= & \frac{1}{2} \int d \mathbf{r}\left\{K_{11}[\boldsymbol{\nabla} \cdot \mathbf{n}]^{2}+K_{22}[\mathbf{n} \cdot(\boldsymbol{\nabla} \times \mathbf{n})]^{2}\right. \\
& \left.+K_{33}[\mathbf{n} \times(\boldsymbol{\nabla} \times \mathbf{n})]^{2}\right\},
\end{aligned}
$$

which has three contributions: the splay, twist, and bend modes. The parameters $K_{\alpha \alpha}(\alpha=1,2,3)$, called Frank elastic constants, control almost exclusively the structure and the properties of nematic liquid crystals at mesoscopic length scales. Expressions that relate them to the microscopic properties of liquid crystals are thus clearly of interest.

Several microscopic approaches have been proposed and employed in the past. ${ }^{758}$ Poniewierski and Stecki ${ }^{35}$ have used the density functional formalism ${ }^{59}$ to derive a set of equations which connects the elastic constants with the direct pair correlation function (DCF), one of the central quantities in liquid state theories. ${ }^{60,61} \mathrm{In}$ a coordinate frame where the $z$ axis points along the director $\mathbf{n}$, the equations read

$$
\begin{aligned}
K_{11}= & \frac{k_{B} T}{2} \int r_{x}^{2} c\left(\mathbf{r}, \mathbf{u}_{1}, \mathbf{u}_{2}\right) \\
& \times \rho^{(1) \prime}\left(u_{1 z}\right) \rho^{(1) \prime}\left(u_{2 z}\right) u_{1 x} u_{2 x} d \mathbf{r} d \mathbf{u}_{1} d \mathbf{u}_{2},
\end{aligned}
$$

$$
\begin{aligned}
K_{22}= & \frac{k_{B} T}{2} \int r_{x}^{2} c\left(\mathbf{r}, \mathbf{u}_{1}, \mathbf{u}_{2}\right) \\
& \times \rho^{(1) \prime}\left(u_{1 z}\right) \rho^{(1) \prime}\left(u_{2 z}\right) u_{1 y} u_{2 y} d \mathbf{r} d \mathbf{u}_{1} d \mathbf{u}_{2}, \\
K_{33}= & \frac{k_{B} T}{2} \int r_{z}^{2} c\left(\mathbf{r}, \mathbf{u}_{1}, \mathbf{u}_{2}\right) \\
& \times \rho^{(1) \prime}\left(u_{1 z}\right) \rho^{(1) \prime}\left(u_{2 z}\right) u_{1 x} u_{2 x} d \mathbf{r} d \mathbf{u}_{1} d \mathbf{u}_{2},
\end{aligned}
$$

where the vector $\mathbf{r}$ connects the centers of mass of two molecules 1 and $2, \mathbf{u}_{1}, \mathbf{u}_{2}$ are unit vectors along the molecule axes, $c\left(\mathbf{r}, \mathbf{u}_{1}, \mathbf{u}_{2}\right)$ denotes the DCF in the nematic liquid, and $\rho^{(1) \prime}\left(u_{z}\right)$ is the derivative of the one-particle distribution function with respect to $u_{z}$. The integrals $\int d \mathbf{r}$ run over all space, and $\int d \mathbf{u}$ over the full solid angle, $T$ is the temperature, and $k_{B}$ is the Boltzmann constant.

Equations of the form (2)-(4) have later been rederived $^{36-46}$ and applied in theories ${ }^{47-58}$ and simulations $^{62-65}$ to study elastic constants in nematic liquid crystals. ${ }^{66}$ The main difficulty with the Poniewierski-Stecki equations is that they depend on the DCF in the nematic phase, which is not known. Theories have resorted to approximations, e.g., they use a DCF from an effectively isotropic reference state, ${ }^{37-42}$ or from a state with perfectly aligned particles..$^{29,30,55}$ Simulation studies ${ }^{62-65}$ have neglected the explicit angular dependence of the pair correlation functions on the orientation of the director. Longa et al. have recently pointed out that this approximation may not be adequate in nematic liquid crystals. ${ }^{67}$

Alternatively, the elastic constants can also be determined directly from the long-wavelength fluctuations of the order tensor in Fourier space

$$
\mathbf{Q}(\mathbf{k})=\frac{V}{N} \sum_{i=1}^{N}\left(\frac{3}{2} \mathbf{u}_{i} \otimes \mathbf{u}_{i}-\frac{1}{2} \mathbf{I}\right) \exp \left(i \mathbf{k} \cdot \mathbf{r}_{i}\right),
$$

where the sum runs over all particles $i$ in the system, I denotes the unit matrix and $\otimes$ the dyadic product of two vectors. The largest eigenvalue of the $3 \times 3$ matrix $\mathbf{Q}$ at zero 
wave vector $\left(\left.\mathbf{Q}(\mathbf{k})\right|_{\mathbf{k}=0}\right)$ is the nematic order parameter $V P_{2}$, and the corresponding eigenvector is the director $\mathbf{n}$ of the nematic liquid.

In a reference frame where the $z$ axis points along $\mathbf{n}$ and the $y$ axis is perpendicular to $\mathbf{k}$, the order tensor fluctuations have the limiting long-wavelength behavior ${ }^{3}$

$$
\begin{aligned}
& \left\langle\left|Q_{x z}(\mathbf{k})\right|^{2}\right\rangle \stackrel{k \rightarrow 0}{\sim} \frac{9}{4} \frac{\left\langle P_{2}\right\rangle^{2} V k_{B} T}{K_{11} k_{x}^{2}+K_{33} k_{z}^{3}}, \\
& \left\langle\left|Q_{y z}(\mathbf{k})\right|^{2}\right\rangle \stackrel{k \rightarrow 0}{\sim} \frac{9}{4} \frac{\left\langle P_{2}\right\rangle^{2} V k_{B} T}{K_{22} k_{x}^{2}+K_{33} k_{z}^{3}} .
\end{aligned}
$$

Provided the simulated systems are sufficiently large, the elastic constants can be extracted directly from Eqs. (6) and (7)..$^{56,68-71}$

Allen et al. ${ }^{71}$ have used this method to study elastic constants in a model liquid crystal, which had already been investigated earlier by Stelzer et al. ${ }^{63,64}$ using the Poniewierski-Stecki equations (2)-(4). The results disagreed by an order of magnitude. Since the determination of elastic constants via Eqs. (6) and (7) is straightforward, it seems reliable and the values calculated by Allen et al. are presumably accurate. On the other hand, Stelzer et al. ${ }^{63,64}$ use an "unoriented nematic approximation," where pair correlation functions are replaced by their average over all orientations of the director. Given the importance of the Poniewierski-Stecki equations, a clearcut test of the applicability of Eqs. (2)-(4) in a nematic liquid crystal is desirable. To the knowledge of the present authors, no one has yet employed the Poniewierski-Stecki equations with the exact DCF of a nematic state. This is presumably due to the fact that no method has been proposed so far which allows one to extract the full orientation dependent DCF from computer simulation data.

The present work attempts to remedy this situation. We propose a way to calculate the DCF without any approximations from a spherical harmonic expansion of the pair distribution function in a uniaxial nematic liquid crystal. The expansion coefficients can be determined from computer simulations in a straightforward manner. ${ }^{61}$ A conveniently reformulated version of Eqs. (2)-(4) then allows one to calculate the Frank elastic constants $K_{11}, K_{22}$, and $K_{33}$ from a direct inspection of expansion coefficients of the DCF in Fourier space. We apply the method to a model system of soft ellipsoidal particles in the nematic phase. For comparison, we also compute the Frank elastic constants from the fluctuations of the order tensor, Eqs. (6) and (7). We find that the values are in good agreement. Our results thus show that the Poniewierski-Stecki theory in combination with the correct DCF can be used to bridge between the microscopic properties of nematic liquid crystals and their mesoscopic, i.e., elastic properties.

Our paper is organized as follows. We develop the theoretical tools needed for our procedure in Sec. II. Section III gives details of the simulation model and the simulation techniques. The results are presented in Sec. IV and discussed in Sec. V.

\section{THEORETICAL BACKGROUND}

We begin by recalling some common definitions. ${ }^{72}$ Let us denote by $\rho(\mathbf{u}, \mathbf{r})$ the local number density of particles with orientation $\mathbf{u}$ at position $\mathbf{r}$. In a uniaxial nematic liquid at equilibrium with director $\mathbf{n}_{0}$, it is distributed according to a one-particle distribution function $\langle\rho(\mathbf{u}, \mathbf{r})\rangle=\rho^{(1)}(\mathbf{u})$, that actually depends on $\left|\mathbf{u} \cdot \mathbf{n}_{0}\right|$ only. The pair distribution function $\rho^{(2)}\left(\mathbf{u}_{1}, \mathbf{u}_{2}, \mathbf{r}_{1}-\mathbf{r}_{2}\right)$ gives the probability of finding a particle with the orientation $\mathbf{u}_{1}$ at the position $\mathbf{r}_{1}$, and another particle with orientation $\mathbf{u}_{2}$ at $\mathbf{r}_{2}$. Particles at infinite distance become uncorrelated, hence $\rho^{(2)}\left(\mathbf{u}_{1}, \mathbf{u}_{2}, \mathbf{r}\right) \stackrel{r \rightarrow \infty}{\rightarrow} \rho^{(1)}$ $\times\left(\mathbf{u}_{1}\right) \rho^{(1)}\left(\mathbf{u}_{2}\right)$. This motivates the definition of the so-called total correlation function

$$
h\left(\mathbf{u}_{1}, \mathbf{u}_{2}, \mathbf{r}\right)=\frac{\rho^{(2)}\left(\mathbf{u}_{1}, \mathbf{u}_{2}, \mathbf{r}\right)}{\rho^{(1)}\left(\mathbf{u}_{1}\right) \rho^{(1)}\left(\mathbf{u}_{2}\right)}-1,
$$

which measures the total effect of a particle 1 on a particle 2 . This effect is often separated into two parts: a hypothetical "direct" effect of 1 on 2, characterized by the direct correlation function $c\left(\mathbf{u}_{1}, \mathbf{u}_{2}, \mathbf{r}\right)$ and an "indirect" effect, where 1 is assumed to influence other particles 3 , 4, etc., which in turn affect 2. The total correlation function is related to the DCF via the Ornstein-Zernike equation ${ }^{60}$

$$
\begin{aligned}
h\left(\mathbf{u}_{1}, \mathbf{u}_{2}, \mathbf{r}_{12}\right)= & c\left(\mathbf{u}_{1}, \mathbf{u}_{2}, \mathbf{r}_{12}\right)+\int c\left(\mathbf{u}_{1}, \mathbf{u}_{3}, \mathbf{r}_{13}\right) \rho^{(1)} \\
& \times\left(\mathbf{u}_{3}\right) h\left(\mathbf{u}_{3}, \mathbf{u}_{2}, \mathbf{r}_{32}\right) d \mathbf{u}_{3} d \mathbf{r}_{3},
\end{aligned}
$$

where $\mathbf{r}_{i j}$ abbreviates $\mathbf{r}_{i}-\mathbf{r}_{j}$.

In the framework of density functional theories, the direct correlation function has another interpretation as the second functional derivative of the excess free energy with respect to local density distortions $\delta \rho(\mathbf{u}, \mathbf{r})=\rho(\mathbf{u}, \mathbf{r})-\rho^{(1)}(\mathbf{u}$ $\left.\cdot \mathbf{n}_{0}\right) .{ }^{60}$ To lowest order in $\delta \rho$, the expansion of the free energy functional about an undistorted equilibrium reference state is given by

$$
\begin{aligned}
\delta^{2} \mathcal{F}= & \frac{k_{B} T}{2} \int\left[\frac{\delta\left(\mathbf{u}_{1}-\mathbf{u}_{2}\right) \delta\left(\mathbf{r}_{12}\right)}{\rho^{(1)}\left(\mathbf{u}_{1} \cdot \mathbf{n}_{0}\right)}-c\left(\mathbf{u}_{1}, \mathbf{u}_{2}, \mathbf{r}_{12}\right)\right] \\
& \times \delta \rho\left(\mathbf{u}_{1}, \mathbf{r}_{1}\right) \delta \rho\left(\mathbf{u}_{2}, \mathbf{r}_{2}\right) d \mathbf{r}_{1} d \mathbf{r}_{2} d \mathbf{u}_{1} d \mathbf{u}_{2} .
\end{aligned}
$$

In systems of particles with uniaxial symmetry, further approximations are not needed. ${ }^{43}$ However, the derivation is greatly simplified by the additional assumption that the relevant long-wavelength distortions can be expressed as local distortions of the director $\mathbf{n}(\mathbf{r})$, and that the density distribution is otherwise at local equilibrium ${ }^{35}$

$$
\rho(\mathbf{u}, \mathbf{r}) \approx \rho^{(1)}(\mathbf{u} \cdot \mathbf{n}(\mathbf{r})) .
$$

Expanding the free energy in terms of $\delta \mathbf{n}(\mathbf{r})=\mathbf{n}(\mathbf{r})-\mathbf{n}_{0}$ rather than $\delta \rho(\mathbf{u}, \mathbf{r})$ and switching to a representation in Fourier space, Eq. (10) then reads

$$
\begin{aligned}
\delta^{2} \mathcal{F}= & \frac{V k_{B} T}{2} \int\left[\frac{\delta\left(\mathbf{u}_{1}-\mathbf{u}_{2}\right)}{\rho^{(1)}\left(\mathbf{u}_{1} \cdot \mathbf{n}_{0}\right)}-c\left(\mathbf{u}_{1}, \mathbf{u}_{2}, \mathbf{k}\right)\right] \\
& \times \rho^{(1) \prime}\left(\mathbf{u}_{1} \cdot \mathbf{n}_{0}\right) \rho^{(1) \prime}\left(\mathbf{u}_{2} \cdot \mathbf{n}_{0}\right) \times\left[\mathbf{u}_{1} \cdot \delta \mathbf{n}(\mathbf{k})\right] \\
& \times\left[\mathbf{u}_{2} \cdot \delta \mathbf{n}(-\mathbf{k})\right] d \mathbf{k} d \mathbf{u}_{1} d \mathbf{u}_{2} .
\end{aligned}
$$


This expression has to be related to Eq. (1), which has the Fourier representation

$$
\begin{aligned}
\mathcal{F}\{\mathbf{n}(\mathbf{k})\}= & \frac{1}{2} \int d \mathbf{k}\left\{K_{11}[\mathbf{k} \cdot \mathbf{n}]^{2}+K_{22}[\mathbf{n} \cdot(\mathbf{k} \times \mathbf{n})]^{2}\right. \\
& \left.+K_{33}[\mathbf{n} \times(\mathbf{k} \times \mathbf{n})]^{2}\right\} .
\end{aligned}
$$

To this end, we expand the DCF $c\left(\mathbf{u}_{1}, \mathbf{u}_{2}, \mathbf{k}\right)$ in Eq. (12) in powers of $\mathbf{k}$ up to second order. For convenience, we choose a coordinate frame such that the $z$ axis points in the direction of $\mathbf{n}_{0}$ (director frame).

Since a global rotation of the director $\mathbf{n}$ does not change the free energy, the leading term $\mathbf{k}=0$ must vanish, i.e., one has

$$
\begin{aligned}
\int \frac{\rho^{(1) \prime}\left(u_{z}\right)^{2}}{\rho^{(1)}\left(u_{z}\right)} u_{\alpha}^{2} d \mathbf{u}= & \int c\left(\mathbf{u}_{1}, \mathbf{u}_{2}, \mathbf{k}=0\right) \rho^{(1) \prime}\left(u_{1, z}\right) \\
& \times \rho^{(1) \prime}\left(u_{2, z}\right) u_{1, \alpha} u_{2, \alpha} d \mathbf{u}_{1} d \mathbf{u}_{2}
\end{aligned}
$$

for $\alpha=x, y$. Equation (14) has been derived in a different context by Gubbins ${ }^{73}$ and is quite generally valid. For symmetry reasons, the terms linear in $\mathbf{k}$ in the expansion of (12) vanish too. The quadratic terms lead to an expression of the form (13), with $K_{i i}$ given by

$$
\begin{aligned}
K_{11}= & -\left.\frac{k_{B} T}{2} \int \frac{\partial^{2} c\left(\mathbf{k}, \mathbf{u}_{1}, \mathbf{u}_{2}\right)}{\partial k_{x}^{2}}\right|_{\mathbf{k}=0} \\
& \times \rho^{(1)^{\prime}}\left(u_{1 z}\right) \rho^{(1)^{\prime}}\left(u_{2 z}\right) u_{1 x} u_{2 x} d \mathbf{u}_{1} d \mathbf{u}_{2}, \\
K_{22}= & -\left.\frac{k_{B} T}{2} \int \frac{\partial^{2} c\left(\mathbf{k}, \mathbf{u}_{1}, \mathbf{u}_{2}\right)}{\partial k_{x}^{2}}\right|_{\mathbf{k}=0} \\
& \times \rho^{(1) \prime}\left(u_{1 z}\right) \rho^{(1) \prime}\left(u_{2 z}\right) u_{1 y} u_{2 y} d \mathbf{u}_{1} d \mathbf{u}_{2}, \\
K_{33}= & -\left.\frac{k_{B} T}{2} \int \frac{\partial^{2} c\left(\mathbf{k}, \mathbf{u}_{1}, \mathbf{u}_{2}\right)}{\partial k_{z}^{2}}\right|_{\mathbf{k}=0}, \\
& \times \rho^{(1) \prime}\left(u_{1 z}\right) \rho^{(1) \prime}\left(u_{2 z}\right) u_{1 x} u_{2 x} d \mathbf{u}_{1} d \mathbf{u}_{2},
\end{aligned}
$$

which is the Fourier space version of the PoniewierskiStecki equations (2)-(4). As mentioned above, the same result can be derived without the approximation (11) for systems of particles with uniaxial symmetry. ${ }^{43}$ Compact expressions for the correction terms in systems of asymmetric molecules have been given by Yokoyama. ${ }^{44}$ In this paper, we shall be concerned with uniaxially symmetric molecules only.

For practical applications, it is convenient to expand all orientation dependent functions in spherical harmonics $Y_{l m}(\mathbf{u})$. In the director frame, we obtain

$$
\rho^{(1)}(\mathbf{u})=\varrho \sum_{l \text { even }} f_{l} Y_{l 0}(\mathbf{u})
$$

where $\varrho$ is the total bulk number density, and

$$
\begin{aligned}
F\left(\mathbf{u}_{1}, \mathbf{u}_{2}, \mathbf{r}\right)= & \sum_{\substack{l_{1}, l_{2}, l \\
m_{1}, m_{2}, m}} F_{l_{1} m_{1} l_{2} m_{2} l m}(r) \\
& \times Y_{l_{1} m_{1}}\left(\mathbf{u}_{1}\right) Y_{l_{2} m_{2}}\left(\mathbf{u}_{2}\right) Y_{l m}(\hat{\mathbf{r}}), \\
F\left(\mathbf{u}_{1}, \mathbf{u}_{2}, \mathbf{k}\right)= & \sum_{l_{1}, l_{2}, l} F_{l_{1} m_{1} l_{2} m_{2} l m}(k) \\
& \times Y_{1}, m_{2}, m \\
&
\end{aligned}
$$

Here $F$ stands for any of $\rho^{(2)}, h$, or $c, \hat{\mathbf{r}}$ denotes the unit vector $\mathbf{r} / r$, and $\hat{\mathbf{k}}$ the unit vector $\mathbf{k} / k$. The symmetry of the nematic phase ensures that all coefficients are real and only coefficients with $m+m_{1}+m_{2}=0$, and $l+l_{1}+l_{2}$ even, enter the expansions (19) and (20). If the molecules have uniaxial symmetry, every single $l_{i}$ has to be even in addition.

Next we derive matrix versions of Eqs. (8) and (9). To simplify the expressions, we introduce the notation

$$
\begin{aligned}
\Gamma_{m m^{\prime} m^{\prime \prime}}^{l l^{\prime} l^{\prime \prime}}= & \int d \mathbf{u} Y_{l m}^{*}(\mathbf{u}) Y_{l^{\prime}, m^{\prime}}(\mathbf{u}), Y_{l^{\prime \prime}, m^{\prime \prime}}(\mathbf{u}) \\
= & \sqrt{\frac{\left(2 l^{\prime \prime}+1\right)\left(2 l^{\prime}+1\right)}{4 \pi(2 l+1)}} C\left(l^{\prime \prime} l^{\prime} l ; 000\right) \\
& \times C\left(l^{\prime \prime} l^{\prime} l ; m^{\prime \prime} m^{\prime} m\right),
\end{aligned}
$$

where $C$ are the Clebsch-Gordan coefficients. The total correlation function $h$ can then be calculated from $\rho^{(2)}$ by inversion of the matrix version of Eq. (8),

$$
\begin{aligned}
\rho_{l_{1} m_{1} l_{2} m_{2} l m}^{(2)}(r)= & \varrho^{2}\left(\sqrt{4 \pi} f_{l_{1}} f_{l_{2}} \delta_{m_{1} 0} \delta_{m_{2} 0} \delta_{l 0} \delta_{m 0}\right. \\
& \left.+\sum_{\substack{l_{1}^{\prime} l_{1}^{\prime \prime} \\
l_{2}^{\prime}, l_{2}^{\prime \prime}}} h_{l_{1}^{\prime} m_{1} l_{2}^{\prime} m_{2} l m}(r) f_{l_{1}^{\prime \prime}} f_{l_{2}^{\prime \prime}} \Gamma_{m_{1} m_{1} l_{1} l_{1}^{\prime} l_{1}^{\prime \prime} \Gamma_{m_{2} m_{2} 0}^{l_{2} l_{2}^{\prime} l_{2}^{\prime \prime}}}\right) .
\end{aligned}
$$

Equation (22) is a linear system of equations and can be solved for the coefficients of $h$ by standard numerical methods.

The Ornstein-Zernike equation (9) is most conveniently solved in Fourier space k. We calculate the coefficients $h_{l_{1} m_{1} l_{2} m_{2} l m}(k)$ of the total correlation function in Fourier space by using the Hankel transformation ${ }^{61}$

$$
h_{l_{1} m_{1} l_{2} m_{2} l m}(k)=4 \pi i^{l} \int_{0}^{\infty} r^{2} j_{l}(k r) h_{l_{1} m_{1} l_{2} m_{2} l m}(r) d r \text {, }
$$

with the spherical Bessel functions $j_{l}$. The matrix version of the Ornstein-Zernike equation (9) in Fourier space reads 


$$
\begin{aligned}
h_{l_{1} m_{1} l_{2} m_{2} l m}(k)= & c_{l_{1} m_{1} l_{2} m_{2} l m}(k) \\
& +\varrho \sum_{\substack{l_{3} l_{3}^{\prime} l_{3}^{\prime \prime} m_{3} \\
l^{\prime} m^{\prime} l^{\prime \prime} m^{\prime \prime}}} c_{l_{1} m_{1} l_{3} m_{3} l^{\prime} m^{\prime}}(k) \\
& \times h_{l_{3}^{\prime} m_{3} l_{2} m_{2} l^{\prime \prime} m^{\prime \prime}}(k) f_{l_{3}^{\prime \prime}} \Gamma_{m m^{\prime} m^{\prime \prime}}^{l l^{\prime} l^{\prime \prime}} \Gamma_{m_{3} m_{3} 0}^{l_{3} l_{3}^{\prime} l_{3}^{\prime \prime}} \\
& \times(-1)^{m_{3}} .
\end{aligned}
$$

The result for the direct correlation function $c(k)$ is readily transformed back into real space by another Hankel transformation. However, this is not necessary for our purpose, because the Poniewierski-Stecki equations assume a very handy form in Fourier space: the spherical harmonic representation of Eqs. (15)-(17) reads

$$
K_{i i}=\left.\frac{1}{2} \frac{d^{2}}{d k^{2}} C_{i i}(k)\right|_{k=0} \text { for } i=1,2,3
$$

with

$$
\begin{aligned}
C_{i i}(k)= & \frac{k_{B} T \varrho^{2}}{8 \sqrt{\pi}} \sum_{l_{1} l_{2}} \sqrt{l_{1}\left(l_{1}+1\right)} \sqrt{l_{2}\left(l_{2}+1\right)} f_{l_{1}} f_{l_{2}} \\
& \times\left\{\left[c_{l_{1} 1 l_{2}-100}(k)+c_{l_{1}-1 l_{2} 100}(k)\right]\right. \\
& +v_{i} \frac{\sqrt{5}}{2}\left[c_{l_{1} 1 l_{2}-120}(k)+c_{l_{1}-1 l_{2} 120}(k)\right] \\
& \left.+w_{i} \frac{\sqrt{15}}{\sqrt{8}}\left[c_{l_{1} 1 l_{2} 12-2}(k)+c_{l_{1}-1 l_{2}-122}(k)\right]\right\}
\end{aligned}
$$

and $\left(v_{1}, v_{2}, v_{3}\right)=(-1,-1,2),\left(w_{1}, w_{2}, w_{3}\right)=(-1,1,0)$. Deriving these equations, we have exploited the relation $\int d \mathbf{r} F(\mathbf{r}) r_{\alpha}^{2}=-\partial^{2} F(\mathbf{k}) /\left.\partial k_{\alpha}^{2}\right|_{\mathbf{k}=0}$ and properties of spherical harmonics. Finally, Eq. (14) can be rewritten as

$$
C_{i i}(k=0)=-k_{B} T \pi \int_{-1}^{1} d u_{z}\left(1-u_{z}^{2}\right) \frac{\rho^{(1) \prime}\left(u_{z}\right)^{2}}{\rho^{(1)}\left(u_{z}\right)},
$$

where $C_{i i}(k)$ is defined as in Eq. (26).

\section{MODEL AND SIMULATION DETAILS}

We performed computer simulations of a system of axially symmetric rigid particles, which interact via a simple repulsive pair potential

$$
V_{i j}=\left\{\begin{array}{l}
4 \epsilon_{0}\left(X_{i j}^{12}-X_{i j}^{6}\right)+\epsilon_{0}: \quad X_{i j}^{6}>1 / 2, \\
0: \quad \text { otherwise. }
\end{array}\right.
$$

Here $X_{i j}=\sigma_{0} /\left(r_{i j}-\sigma_{i j}+\sigma_{0}\right), r_{i j}$ denotes the distance between particles $i$ and $j$, and the shape function

$$
\begin{aligned}
\sigma_{i j}\left(\mathbf{u}_{i}, \mathbf{u}_{j}, \hat{\mathbf{r}}_{i j}\right)= & \sigma_{0}\left\{1-\frac{\chi}{2}\left[\frac{\left(\mathbf{u}_{i} \cdot \hat{\mathbf{r}}_{i j}+\mathbf{u}_{j} \cdot \hat{\mathbf{r}}_{i j}\right)^{2}}{1+\chi \mathbf{u}_{i} \cdot \mathbf{u}_{j}}\right.\right. \\
& \left.\left.+\frac{\left(\mathbf{u}_{i} \cdot \hat{\mathbf{r}}_{i j}-\mathbf{u}_{j} \cdot \hat{\mathbf{r}}_{i j}\right)^{2}}{1-\chi \mathbf{u}_{i} \cdot \mathbf{u}_{j}}\right]\right\}^{-1 / 2},
\end{aligned}
$$

approximates the contact distance between two ellipsoids of elongation $\kappa=\sigma_{\text {end-end }} / \sigma_{\text {side-side }}=\sqrt{(1+\chi) /(1-\chi)}$ with orientations $\mathbf{u}_{i}$ and $\mathbf{u}_{j}$, which are separated by a centercenter vector in the direction of $\hat{\mathbf{r}}_{i j}=\mathbf{r}_{i j} / r_{i j}{ }^{74}$ We use throughout scaled units defined in terms of $\epsilon_{0}, \sigma_{0}$, the particle mass $m_{0}$ and the Boltzmann constant $k_{B}$. We studied systems of particles with elongation $\kappa=3$ at temperature $T$ $=0.5$ and number density $\varrho=0.3$. The pressure was $P$ $=2.60{ }^{75}$ This corresponds to a state well in the nematic phase: at fixed temperature $T=0.5$, the fluid remains nematic down to the density $\varrho=0.29$ or, equivalently, the pressure $P=2.35$. $^{76}$ The average order parameter density in our system was $\left\langle P_{2}\right\rangle=0.69$ and the fourth rank parameter was $\left\langle P_{4}\left(\mathbf{u} \cdot \mathbf{n}_{0}\right)\right\rangle=0.31, \quad P_{4}(x)=\left(35 x^{4}-30 x^{2}+3\right) / 8$ being the fourth Legendre polynomial.

The pair distribution function was determined in systems of $N=1000,4000$, and 8000 particles in cubic boxes with periodic boundary conditions. For the $N=1000$ system we used a Monte Carlo (MC) program by Lange. ${ }^{76}$ Trial moves picked a particle at random and attempted in random order either a rotation or a translation, with maximum step sizes chosen such that the Metropolis acceptance rate was roughly $30 \%$. The larger systems were studied with a massively parallel computer, using a domain decomposition molecular dynamics (MD) program, that has been codeveloped by one of us (G.G.). These simulations were performed in the microcanonical ensemble using the RATTLE integrator ${ }^{77,78}$ with time step $\Delta t=0.003$ (Ref. 79) and molecular moment of inertia $I=2.5$. Run lengths were 8 million MC steps, one MC step consisting of $2 \mathrm{~N}$ trial moves, or 10 million MD steps, respectively; data for the pair distribution function were collected every 1000 or 10000 steps.

The order tensor fluctuations are sampled most efficiently if the $\mathbf{k}$ vectors in Eqs. (6) and (7) are always on the same grid. They were therefore determined from independent simulations in an ensemble where the director $\mathbf{n}_{0}$ was constrained to the $Z$ axis of the simulation box. ${ }^{71}$ Thus the $x y z$ frame of Eqs. (6) and (7) becomes coincident with the $X Y Z$ frame of the simulation box. The constraint was implemented in the MD simulations by adding two global Lagrange multipliers to the integrator, so that $Q_{X Z}(0)$ $=Q_{Y Z}(0)=0$ at every time step. Our procedure was similar to that introduced by Allen et al., ${ }^{71}$ except that we used an improved integrator ${ }^{80}$ designed in the spirit of RATTLE, ${ }^{77,78}$ so that it is symplectic and fulfills the constraints exactly. The same integrator has already been used ${ }^{81}$ to calculate $K_{22}$ in a Gay-Berne fluid $;^{82}$ the value compared well with an estimate from a thermodynamic perturbation approach. Here, we simulated a system of $N=4000$ particles in a cubic box over 10 million MD steps, and a system of $N=16000$ particles in an elongated box with side ratios $L_{X}: L_{Y}: L_{Z}=1: 1: 2$ (Ref. $83)$ over 5 million MD steps. Data for the order tensor were collected every 200 steps. The largest autocorrelation times were of the order of $10^{5} \mathrm{MD}$ steps at the lowest $k$ values and dropped rapidly below $1000 \mathrm{MD}$ steps for higher $k$.

\section{DATA ANALYSIS AND RESULTS}

We begin by presenting the results for the order tensor fluctuations. Following Ref. 71, we calculated the quantities 

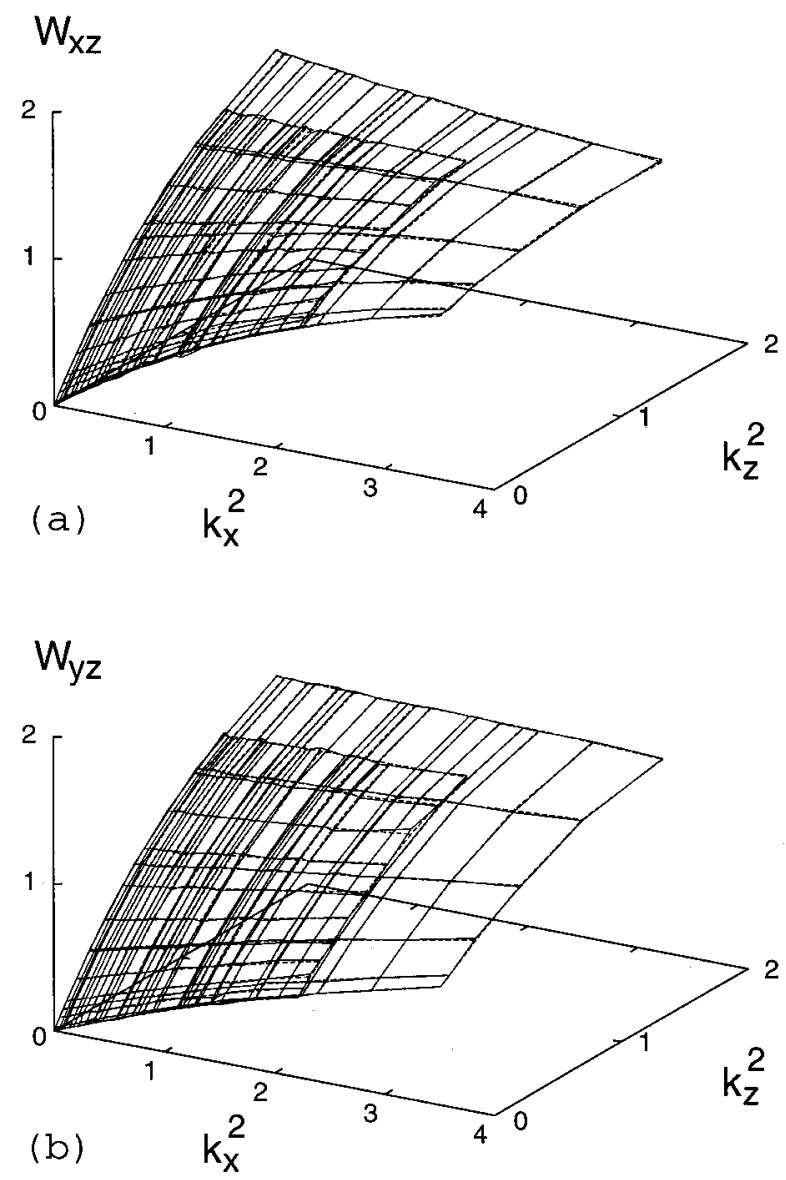

FIG. 1. $\mathcal{W}_{x z}$ (a) and $\mathcal{W}_{y z}$ (b) surfaces for $N=4000$ (cubic box) and $N$ $=16000$ (elongated box); the smaller, finer spaced grids correspond to the larger system. The fits (dotted lines) coincide almost perfectly with the data (solid lines).

$$
\begin{aligned}
& \mathcal{W}_{x z}(\mathbf{k})=\frac{9\left\langle P_{2}\right\rangle^{2} V k_{B} T}{4\left\langle\left|Q_{x z}(\mathbf{k})\right|^{2}\right\rangle} \stackrel{k \rightarrow 0}{\sim} K_{11} k_{x}^{2}+K_{33} k_{z}^{2}, \\
& \mathcal{W}_{y z}(\mathbf{k})=\frac{9\left\langle P_{2}\right\rangle^{2} V k_{B} T}{4\left\langle\left|Q_{y z}(\mathbf{k})\right|^{2}\right\rangle} \stackrel{k \rightarrow 0}{\sim} K_{22} k_{x}^{2}+K_{33} k_{z}^{2},
\end{aligned}
$$

where the frame is chosen such that $\mathbf{k}$ lies in the $x z$ plane [cf. Eqs. (6) and (7)]. More specifically, we evaluated the order tensor in Fourier space $\mathbf{Q}(\mathbf{k})$ on a $\mathbf{k}$ grid with $6 \times 6 \times 6$ grid points in the small system $(N=4000)$, and $6 \times 6 \times 12$ grid points in the large system $(N=16000)$. Then we applied a rotation $\mathbf{Q}^{(x y z)}(\mathbf{k})=\mathbf{U}(\mathbf{k}) \mathbf{Q}^{(X Y Z)}(\mathbf{k}) \mathbf{U}^{T}(\mathbf{k})$ into the desired coordinate frame such that $k_{y}=0$, and calculated the averages $\left\langle\left|\mathbf{Q}_{\alpha z}(\mathbf{k})\right|^{2}\right\rangle$ and the $\mathcal{W}_{\alpha z}(\mathbf{k})$ surface in that frame. Because of the constraint on $\mathbf{n}_{0}, \mathbf{U}(\mathbf{k})$ is a constant throughout the run.

In the high wavelength limit $k \rightarrow \infty, \mathcal{W}_{\alpha z}(\mathbf{k})(\alpha=1,2)$ takes the value ${ }^{71}$

$$
\mathcal{W}_{\alpha z}(\mathbf{k}) \stackrel{{ }^{k \rightarrow \infty}}{\longrightarrow} \frac{\left\langle P_{2}\right\rangle^{2} \rho k_{B} T}{\left\langle P_{2}\right\rangle / 21-4\left\langle P_{4}\right\rangle / 35+1 / 15} .
$$

In our simulations, we obtained 1.13 , which is in good agreement with the theoretical value 1.12.

The results for the $\mathcal{W}_{\alpha z}(\mathbf{k})$ surfaces are shown in Fig. 1. The data for the small system (coarse grid) match almost exactly those for the large system (fine grid). They were
TABLE I. Elastic constants from the analysis of order tensor fluctuations for systems of different size $N$.

\begin{tabular}{cccc}
\hline \hline \multirow{2}{*}{$\begin{array}{c}\text { System } \\
\text { size }\end{array}$} & \multicolumn{3}{c}{ Order tensor fluctuations } \\
\cline { 2 - 4 } & $\left\langle K_{11}\right\rangle$ & $\left\langle K_{22}\right\rangle$ & $\left\langle K_{33}\right\rangle$ \\
\hline 4000 & $0.53 \pm 0.01$ & $0.30 \pm 0.01$ & $1.60 \pm 0.01$ \\
16000 & $0.53 \pm 0.01$ & $0.30 \pm 0.01$ & $1.59 \pm 0.01$ \\
\hline \hline
\end{tabular}

fitted to a fourth order polynomial in $k_{x}^{2}$ and $k_{z}^{2}$ (i.e., with highest order terms $\left.k_{x}^{8}, k_{x}^{6} k_{z}^{2}, \ldots, k_{z}^{8}\right)$ without a zeroth order term. Higher orders were disregarded because the fourth order coefficients turned out to be already very small. Normal equations and singular value decomposition gave the same results. Figure 1 demonstrates that the fit is almost perfect. The leading coefficients give the elastic constants, shown in Table I. As expected for elongated molecules, one finds that $K_{33}$ is largest, followed by $K_{11}$ and $K_{22}$.

Next we discuss the results for the pair correlation functions. The spherical harmonics expansion coefficients of the pair distribution function $\rho^{(2)}$ were determined using ${ }^{84}$

$$
\begin{aligned}
& \rho_{l_{1} m_{1} l_{2} m_{2} l m}^{(2)}(r) \\
& \quad=4 \pi \varrho^{2} g(r)\left\langle Y_{l_{1} m_{1}}^{*}\left(\mathbf{u}_{1}\right) Y_{l_{2} m_{2}}^{*}\left(\mathbf{u}_{2}\right) Y_{l m}^{*}(\hat{\mathbf{r}})\right\rangle_{\delta r},
\end{aligned}
$$

where $\langle\cdot\rangle_{\delta r}$ denotes the average over all molecules in a shell $\delta r$ from $r$ to $r+\delta r$, and the function $g(r)$ is the number of molecular centers at distance $r$ from a given molecular center, divided by the number at the same distance in an ideal gas at the same density. The calculation of these averages is very time consuming, since a great number of coefficients has to be evaluated, and was therefore carried out in part on a massively parallel machine. We have determined coefficients for values of $l, l_{i}$ up to $l_{\max }=6$ in all systems, and for values up to $l_{\max }=8$ in the smallest system. The bin size was $\delta r=0.04$ and the cutoff distance $r_{\max }$ was chosen to be $40 \%$ of the box side $L$ in order to reduce boundary effects. ${ }^{85}$

From the pair distribution function we calculated the total correlation function by inverting Eq. (22). The latter was then Fourier transformed according to Eq. (23). There is a subtle problem here: due to the elasticity of the nematic phase, the total correlation function decays algebraically like $1 / r$. This follows directly from Eq. (1). ${ }^{3}$ Before applying Eq. (23), we thus fitted the simulation data points at the largest distances $r>r_{0}$ to a power law of the form $b / r$ and extrapolated $h(r)$ to infinity. ${ }^{86}$ The parameter $r_{0}$ was chosen to be 2.8, 4.0, and 5.3 in systems of $N=1000,4000$, and 8000 particles, respectively.

It turned out that the long-range tail was quite pronounced for coefficients of $h$ with $m_{1}= \pm 1, m_{2}= \pm 1$, and almost negligible for the others. In Fig. 2 we show an example of a coefficient with a pronounced long-range tail, the coefficient $h_{l_{1} m_{1} l_{2} m_{2} l m}(r)$ with $l_{1}=l_{2}=l=2, m_{1}=1, m_{2}$ $=-1$ and $m=0$. The data for different system sizes $N$ $=1000, N=4000$, and $N=8000$ lie almost on top of each other, hence the form of $h(r)$ at $r<r_{\max }$ is not affected by 


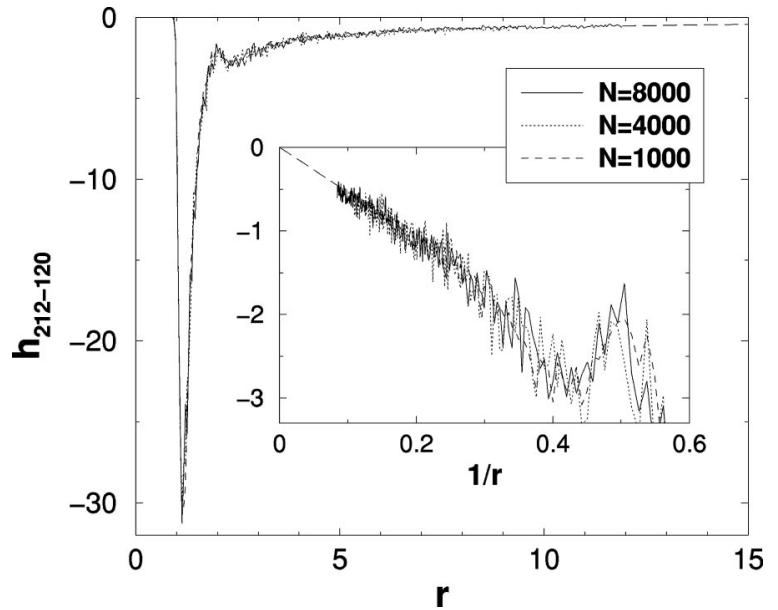

FIG. 2. Expansion coefficient $h_{212-120}(r)$ of the total correlation function $h$ vs $r$ in systems of size $N=8000$ (solid line), $N=4000$ (dotted line), and $N=1000$ (dashed line). Cutoff radii were $r_{\max }=11.9,9.4$, and 6.6, respectively. The long dashed line indicates the extrapolation towards $r \rightarrow \infty$ (for the dataset $N=1000$ ). Inset shows same data vs $1 / r$.

noticeable finite size effects. The dominating finite size problem comes from the uncertainty of the extrapolation, if the available range of $h(r)$ is too short.

The rest of the analysis was straightforward. From the coefficients of the total correlation function in Fourier space, $h_{l_{1} m_{1} l_{2} m_{2} l m}(k)$, those of the DCF were obtained by solving the linear matrix equation (24). Then we calculated the functions $C_{i i}(k)$ as defined in Eq. (26). According to Eq. (25), the elastic constants $K_{i i}$ can be determined from the initial slopes in a plot of $C_{i i}(k)$ versus $k^{2}$. Data for $C_{i i}(k)$ are shown for different system sizes in Fig. 3. The points at zero wave vector $C_{i i}(0)$ were calculated using Eq. (27). They fit nicely on the straight lines at $k \rightarrow 0$, hence the data are consistent with the requirement (14) or (27). This gave additional confidence in the quality of the analysis. The slopes of the straight lines yield the elastic constants.

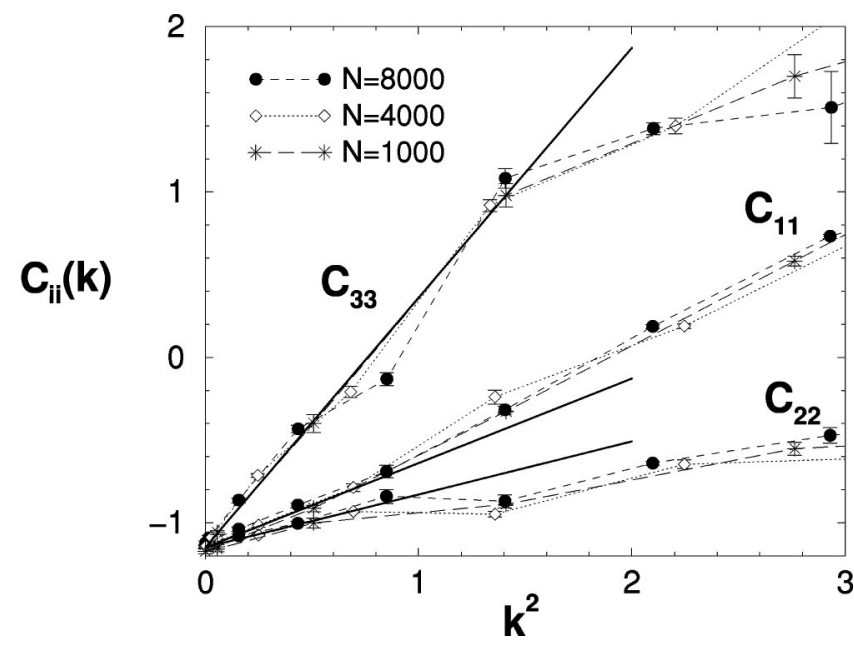

FIG. 3. Weighted sum of the DCF expansion coefficients $C_{i i}(k)$ as defined in Eq. (26) vs $k^{2}$ for different system sizes $N$ (unconstrained director, evaluated using coefficients up to $l_{\max }=6$ ). The points at $k=0$ are taken from Eq. (27). The initial slopes give the elastic constants $K_{i i}$. Thick solid lines indicate corresponding fits for the $N=4000$ system.
TABLE II. Elastic constants from the DCF method for systems of different size $N$. (*) marks a system that has been simulated with a director constraint. Results are shown for different choices of the cutoff value $l_{\max }$ in the spherical harmonics expansion of the pair distribution function $\rho^{(2)}$. See Sec. IV for details.

\begin{tabular}{ccccc}
\hline \hline \multirow{2}{*}{$\begin{array}{c}\text { System } \\
\text { size }\end{array}$} & \multicolumn{4}{c}{ Direct correlation function } \\
\cline { 2 - 5 } 1000 & $l_{\max }$ & $\left\langle K_{11}\right\rangle$ & $\left\langle K_{22}\right\rangle$ & $\left\langle K_{33}\right\rangle$ \\
\hline \multirow{2}{*}{$100.55 \pm 0.02$} & $0.35 \pm 0.03$ & $1.56 \pm 0.04$ \\
& 6 & $0.51 \pm 0.02$ & $0.34 \pm 0.03$ & $1.52 \pm 0.04$ \\
& 4 & $0.53 \pm 0.03$ & $0.23 \pm 0.02$ & $1.32 \pm 0.04$ \\
& 2 & $0.51 \pm 0.01$ & $0.20 \pm 0.01$ & $1.56 \pm 0.04$ \\
4000 & 6 & $0.51 \pm 0.02$ & $0.31 \pm 0.01$ & $1.51 \pm 0.03$ \\
& 4 & $0.65 \pm 0.02$ & $0.27 \pm 0.02$ & $1.23 \pm 0.03$ \\
& 2 & $0.53 \pm 0.01$ & $0.22 \pm 0.01$ & $1.46 \pm 0.03$ \\
$* 4000$ & 6 & $0.52 \pm 0.02$ & $0.31 \pm 0.01$ & $1.51 \pm 0.03$ \\
& 4 & $0.65 \pm 0.02$ & $0.27 \pm 0.02$ & $1.24 \pm 0.04$ \\
& 2 & $0.53 \pm 0.01$ & $0.22 \pm 0.01$ & $1.48 \pm 0.03$ \\
8000 & 6 & $0.51 \pm 0.02$ & $0.33 \pm 0.02$ & $1.48 \pm 0.03$ \\
& 4 & $0.61 \pm 0.01$ & $0.29 \pm 0.02$ & $1.25 \pm 0.04$ \\
& 2 & $0.54 \pm 0.01$ & $0.23 \pm 0.01$ & $1.47 \pm 0.04$ \\
\hline \hline
\end{tabular}

The results are summarized in Table II. We have calculated the DCF from the pair distribution function $\rho^{(2)}$ using an upper cutoff $l_{\max }=2,4$, and 6 , respectively, in the matrix equations (22) and (24). Already the lowest order calculation with $l_{\max }=2$ gave elastic constants of the correct order of magnitude. Quantitatively reliable results were obtained with $l_{\max } \geqslant 6$ : we checked in the smallest system that the results from calculations with $l_{\max }=6$ and $l_{\max }=8$ do not differ significantly.

Since the calculations with $l_{\max }=8$ were very time consuming (one has 1447 different expansion coefficients), we used $l_{\max }=6$ in the analyses of the larger systems (469 different expansion coefficients).

The results were the same for systems of size $N$ $=1000,4000$, and 8000. Furthermore, they were not affected by the presence of a director constraint: as mentioned in Sec. III, the DCF was mostly calculated in unconstrained systems, but we also studied the DCF in one constrained system for comparison.

Finally, we compare the values of the elastic constants calculated by the DCF approach with those obtained from the order fluctuation analysis, shown in Table I. The values for $K_{11}$ and $K_{22}$ are identical for both methods. $K_{33}$ is slightly underestimated by the DCF analysis with $l_{\max }=6$, but the result increases with $l_{\max }$, and agrees within the error with that of the order fluctuation analysis at $l_{\max }=8$.

One might ask how much the successive coefficients of the (correct) DCF contribute to the elastic constants. We found that the contribution of the coefficients with $l_{1}>4, l_{2}$ $>4$ or $l>4$ is very small. If we include only terms up to $l, l_{i}=4$ in Eq. (26), we obtain $K_{11}=0.55, K_{22}=0.21$, and $K_{33}=1.51$ (in the largest system $N=8000$ ), which is very close to the final values quoted in Table II. However, we could not push this analysis further. If we include only terms up to $l, l_{i}=2$, the resulting $C_{i i}(k)$ are very concave and have no well-defined initial slope in a plot versus $k^{2}$. Hence the 
contributions of successive $l s$ to the elastic constants cannot be distinguished.

\section{SUMMARY AND CONCLUSIONS}

We have presented a method which allows one to determine without approximations the direct correlation functions in nematic liquid crystals from computer simulations, and to calculate elastic constants on that basis according to the Poniewierski-Stecki theory ${ }^{35}(2)-(4)$. We have applied this method to a nematic fluid of soft ellipsoids. In the same system, the elastic constants were also determined by an established approach, the analysis of order tensor fluctuations.

Our study represents a direct test of the PoniewierskiStecki theory. We found that the results obtained with the two methods agree well with each other. The PoniewierskiStecki theory can thus be employed to calculate elastic constants, at least in our system, provided that the exact direct correlation functions are used in the equations.

Hence we have established an alternative way of calculating elastic constants in nematic liquid crystals. As long as a simulation is performed solely to determine elastic constants, the order tensor fluctuation approach is still more efficient: the statistical error of pair correlation functions must be quite small for a reliable DCF analysis, and the analysis is very time consuming. However, the DCF approach has the advantage of being straightforward; elastic constants can be computed from arbitrary bulk simulations, if the pair distribution functions are known with sufficient accuracy. Even the calculation of spatially varying elastic constants, e.g., in the vicinity of surfaces, is conceivable.

The direct correlation function is a central quantity in liquid state theories. The study of direct correlation functions in the nematic phase is therefore interesting in its own right. We shall examine them in more detail and compare them to those in the isotropic phase in a forthcoming publication. ${ }^{87}$

\section{ACKNOWLEDGMENTS}

The authors thank M. P. Allen and H. Lange for fruitful interactions, and P. Teixeira for helpful comments on the paper. The simulations and the analyses were run in part on a CRAY T3E of the HLRZ in Jülich. Two of the authors (N.H.P. and G.G.) received financial support from the German Science Foundation (DFG). The parallel MD program GBMEGA used in this work was originally developed by the EPSRC Complex Fluids Consortium, UK.

${ }^{1}$ P.-G. de Gennes and J. Prost, The Physics of Liquid Crystals (Oxford University Press, Oxford, 1995).

${ }^{2}$ S. Chandrasekhar, Liquid Crystals (Cambridge University Press, Cambridge, 1992).

${ }^{3}$ D. Forster, Hydrodynamic fluctuations, broken symmetry and correlation functions, Frontiers in Physics (Benjamin, Reading, MA, 1975), Vol. 47.

${ }^{4}$ P. M. Chaikin and T. C. Lubensky, Principles of Condensed Matter Physics (Cambridge University Press, Cambridge, 1995).

${ }^{5}$ C. Oseen, Trans. Faraday Soc. 29, 883 (1933); H. Zöcher, ibid. 29, 945 (1933).

${ }^{6}$ F. C. Frank, Discuss. Faraday Soc. 25, 19 (1958).

${ }^{7}$ T. C. Lubensky, Phys. Lett. A 33, 202 (1970).

${ }^{8}$ J. Nehring and A. Saupe, J. Chem. Phys. 54, 337 (1971); 54, 337 (1971).

${ }^{9}$ R. G. Priest, Mol. Cryst. Liq. Cryst. 17, 129 (1972); Phys. Rev. A 7, 720 (1973).
${ }^{10}$ J. P. Straley, Phys. Rev. A 8, 2181 (1973).

${ }^{11}$ T. E. Faber, Proc. R. Soc. London 353, 261 (1977).

${ }^{12}$ D. A. Dunmur and W. H. Miller, Chem. Phys. Lett. 86, 353 (1982).

${ }^{13}$ Th. W. Ruijgrok and K. Sokalski, Physica A 111, 45 (1982).

${ }^{14}$ B. W. Van Der Meer, F. Postma, A. J. Dekker, and W. H. De Jeu, Mol. Phys. 45, 1227 (1982).

${ }^{15}$ W. M. Gelbart and A. Ben-Shaul, J. Chem. Phys. 77, 916 (1982).

${ }^{16}$ S. Sarkar and R. J. A. Tough, J. Phys. I 43, 1543 (1982).

${ }^{17}$ G. Vertogen, S. D. P. Flapper, and C. Dullemond, J. Chem. Phys. 76, 616 (1982).

${ }^{18}$ G. Vertogen, Phys. Lett. A 89, 448 (1982).

${ }^{19}$ E. Govers and G. Vertogen, Liq. Cryst. 2, 31 (1987).

${ }^{20}$ E. Govers and G. Vertogen, Physica A 150, 1 (1988).

${ }^{21}$ E. Govers and G. Vertogen, Liq. Cryst. 5, 323 (1989).

${ }^{22}$ B. Tjipto-Margo and D. E. Sullivan, J. Chem. Phys. 88, 6620 (1988).

${ }^{23}$ S.-W. Lo and R. A. Pelcovits, Phys. Rev. E 42, 4756 (1990).

${ }^{24}$ G. Marrucci and F. Greco, Mol. Cryst. Liq. Cryst. 206, 17 (1991).

${ }^{25}$ A. V. Zakharov, Physica A 175, 327 (1991).

${ }^{26}$ A. V. Zakharov and S. Romano, Phys. Rev. E 58, 7428 (1998).

${ }^{27}$ A. V. Zakharov and R. J. Dong, Phys. Rev. E 630, 1704 (2001).

${ }^{28}$ R. G. Petschek and E. M. Terentjev, Phys. Rev. E 45, 930 (1992).

${ }^{29}$ M. A. Osipov and S. Hess, Mol. Phys. 78, 1191 (1993).

${ }^{30}$ M. A. Osipov and S. Hess, Liq. Cryst. 16, 845 (1994).

${ }^{31}$ L. R. Evangelista, I. Hibler, and A. J. Palangana, Nuovo Cimento D 18, 33 (1996).

${ }^{32}$ L. R. Evangelista, I. Hibler, and H. Mukai, Phys. Rev. E 58, 3245 (1998).

${ }^{33}$ P. A. de Castro, A. J. Palangana, and L. R. Evangelista, Phys. Rev. E 60, 6195 (1999).

${ }^{34}$ T. Sato and A. Teramoto, Macromolecules 29, 4107 (1996).

${ }^{35}$ A. Poniewierski and J. Stecki, Mol. Phys. 38, 1931 (1979).

${ }^{36}$ A. Poniewierski and J. Stecki, Phys. Rev. A 25, 2368 (1982).

${ }^{37}$ Y. Singh, Phys. Rev. A 30, 583 (1984); Liq. Cryst. 2, 31 (1987).

${ }^{38}$ Y. Singh and K. Singh, Phys. Rev. A 33, 3481 (1986).

${ }^{39}$ Y. Singh, S. Singh, and K. Rajesh, Phys. Rev. E 45, 974 (1992).

${ }^{40}$ S. Singh, Phys. Rep. 277, 284 (1996).

${ }^{41}$ M. D. Lipkin, S. A. Rice, and U. Mohanty, J. Chem. Phys. 82, 472 (1985).

${ }^{42}$ P. I. C. Teixeira, V. M. Pergamenshchik, and T. J. Sluckin, Mol. Phys. 80, 1339 (1993).

${ }^{43}$ A. M. Somoza and P. Tarazona, Mol. Phys. 72, 911 (1991).

${ }^{44}$ H. Yokoyama, Phys. Rev. E 55, 2938 (1997).

${ }^{45}$ A. Kapanowski, Phys. Rev. E 55, 7090 (1997).

${ }^{46}$ L. Longa, J. Stelzer, and D. Dunmur, J. Chem. Phys. 109, 1555 (1998).

${ }^{47}$ J. Stecki and A. Poniewierski, Mol. Phys. 41, 1451 (1980).

${ }^{48}$ A. Poniewierski and R. Holyst, Phys. Rev. E 41, 6871 (1990).

${ }^{49}$ V. P. Veshnev and I. I. Ptichkin, Kristallografiya 34, 1217 (1989).

${ }^{50}$ K. Singh and Y. Singh, Phys. Rev. A 34, 548 (1986); 35, 3535 (1987).

${ }^{51}$ J. Ram and Y. Singh, Phys. Rev. E 44, 3718 (1991).

${ }^{52}$ S. Singh, Liq. Cryst. 20, 797 (1996).

${ }^{53}$ T. K. Lahiri, K. Rajesh, and S. Singh, Liq. Cryst. 22, 575 (1997).

${ }^{54}$ K. Singh and N. S. Pandey, Liq. Cryst. 25, 411 (1998).

${ }^{55}$ A. M. Somoza and T. Tarazona, Phys. Rev. A 40, 6069 (1989).

${ }^{56}$ B. Tjipto-Margo, G. T. Evans, M. P. Allen, and D. Frenkel, J. Phys. Chem. 96, 3942 (1992).

${ }^{57}$ Z. D. Zhang and G. C. Yang, J. Mater. Sci. Technol. 15, 377 (1999).

${ }^{58}$ M. F. Holovko and T. G. Sokolovska, J. Mol. Liq. 82, 161 (1999).

${ }^{59}$ R. Evans, in Fundamentals of Inhomogeneous Fluids, edited by D. Henderson (Marcel Dekker, New York, 1992), p. 86.

${ }^{60}$ J. P. Hansen and I. R. McDonald, Theory of Simple Liquids (Academic, London, 1986).

${ }^{61}$ C. G. Gray and K. E. Gubbins, Theory of Molecular Fluids (Oxford University Press, Oxford, 1984), Vol. 1.

${ }^{62}$ J. Stelzer, L. Longa, and H. R. Trebin, J. Chem. Phys. 103, 3098 (1995); 107, 1295 (1997).

${ }^{63}$ J. Stelzer, L. Longa, and H. R. Trebin, Mol. Cryst. Liq. Cryst. 262, 455 (1995).

${ }^{64}$ J. Stelzer, M. A. Bates, L. Longa, and G. R. Luckhurst, J. Chem. Phys. 107, 7483 (1997).

${ }^{65}$ A. V. Zakharov and A. Maliniak, Eur. Phys. J. E 4, 85 (2001).

${ }^{66}$ There are differences, however: In Refs. 37-42, the direct correlation function in the equations is that of an isotropic reference state, whereas in the original Poniewierski-Stecki equations it is that of the nematic phase. The expressions derived by Singh (Ref. 37) differ from the PoniewierskiStecki equations, but they can be cast in the form (2)-(4) if one exploits the symmetry of the reference state. 
${ }^{67}$ L. Longa, G. Cholewiak, R. Trebin, and G. R. Luckhurst, Eur. Phys. J. E 4, 51 (2001).

${ }^{68}$ M. P. Allen and D. Frenkel, Phys. Rev. A 37, 1813 (1988); 42, 3641 (1990).

${ }^{69}$ M. P. Allen and D. J. Cleaver, Phys. Rev. E 43, 1918 (1991).

${ }^{70}$ M. P. Allen and A. J. Masters, Mol. Phys. 79, 277 (1993).

${ }^{71}$ M. P. Allen, M. A. Warren, M. R. Wilson, A. Sauron, and W. Smith, J. Chem. Phys. 105, 2850 (1996).

${ }^{72}$ C. Zannoni, in Advances in the Computer Simulations of Liquid Crystals, NATO Science Series C Vol. 545, edited by P. Pasini and C. Zannoni (Kluwer Academics, Dordrecht, 2000), p. 17.

${ }^{73}$ K. E. Gubbins, Chem. Phys. Lett. 76, 329 (1980).

${ }^{74}$ B. J. Berne and P. Pechukas, J. Chem. Phys. 56, 4213 (1975).

${ }^{75}$ The pressure was determined from the simulation data. The temperature was a fixed parameter in the Monte Carlo simulations. The molecular dynamics simulations were carried out in the microcanonical ensemble with the total energy adjusted such that the temperature had the desired value. Statistical errors are in the third decimal place.

${ }^{76}$ H. Lange, Ph.D. thesis, Universität Mainz (2001).
${ }^{77}$ H. C. Andersen, J. Comput. Phys. 52, 24 (1983).

${ }^{78}$ M. P. Allen and D. J. Tildesley, Computer Simulations of Liquids (Oxford University Press, Oxford, 1989).

${ }^{79}$ In our scaling, the time unit is $\sqrt{m_{0} / \epsilon_{0}} \sigma_{0}$.

${ }^{80} \mathrm{G}$. Germano (in preparation).

${ }^{81}$ G. Germano and M. P. Allen (in preparation).

${ }^{82}$ J. G. Gay and B. J. Berne, J. Chem. Phys. 74, 3316 (1981).

${ }^{83}$ The choice of an elongated box was motivated by the fact that $K_{33}$ is about twice as large as $K_{11}$ and $K_{22}$. Equations (6), (7) or (30), (31) reveal that it is of advantage to work on a $\mathbf{k}$-grid, which is finer in the $z$ direction than in the other two.

${ }^{84}$ W. B. Streett and D. J. Tildesley, Proc. R. Soc. London, Ser. A 348, 485 (1975).

${ }^{85}$ L. R. Pratt and S. W. Haan, J. Chem. Phys. 74, 1873 (1980).

${ }^{86}$ For a few coefficients, it was also necessary to shift the data by a constant. See Ref. 87 for a more detailed presentation of the data analysis.

${ }^{87}$ N. H. Phuong and F. Schmid (in preparation). 